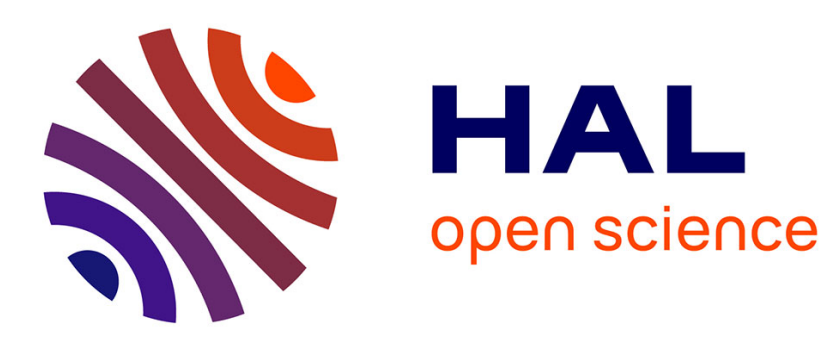

\title{
Aphrodite à Délos : culte privé et public à l'époque hellénistique
}

Cécile Durvye

\section{To cite this version:}

Cécile Durvye. Aphrodite à Délos: culte privé et public à l'époque hellénistique. Revue des Etudes Grecques, 2006, 119 (1), pp.83 - 113. 10.3406/reg.2006.4647 . halshs-01722498

\section{HAL Id: halshs-01722498 \\ https://shs.hal.science/halshs-01722498}

Submitted on 4 Mar 2018

HAL is a multi-disciplinary open access archive for the deposit and dissemination of scientific research documents, whether they are published or not. The documents may come from teaching and research institutions in France or abroad, or from public or private research centers.
L'archive ouverte pluridisciplinaire HAL, est destinée au dépôt et à la diffusion de documents scientifiques de niveau recherche, publiés ou non, émanant des établissements d'enseignement et de recherche français ou étrangers, des laboratoires publics ou privés. 


\title{
Cécile Durvye
}

\section{Aphrodite à Délos : culte privé et public à l'époque hellénistique}

In: Revue des Études Grecques, tome 119, Janvier-juin 2006. pp. 83-113.

\begin{abstract}
This paper was presented the 5th December 2005 at the Association des Etudes grecques. Its purpose is to offer a synthesis of our knowledge concerning the cult of Aphrodite at Delos during the Hellenistic times. This cult has three main forms. A public cult including festivals, the Aphrodisia, is practised during all Delos Independence (314-167 BC). Another cult characterized by a strong familial dimension is created at the end of the 4th century by a Delian named Stesileos who founded a sanctuary ; during the time of Delos Independence, the founder's descendants maintain the cult. We don't know if the public cult survives in the period of the Athenian domination, but the sanctuary of Stesileos develops and welcomes numerous and quite different worshippers.
\end{abstract}

\section{Résumé}

Cette communication, prononcée le 5 décembre 2005 lors d'une séance de l'Association des Études grecques, a pour objet de proposer une synthèse de nos connaissances sur le culte d'Aphrodite à Délos à l'époque hellénistique. Ce culte revêt trois formes principales. Un culte public donnant lieu à des fêtes, les Aphrodisia, est pratiqué pendant toute la période de I'Indépendance délienne (314-167 av. J.-C). Parallèlement, un culte présentant de forts caractères familiaux est fondé à la fin du ive siècle par un Délien nommé Stèsiléos ; pendant l'Indépendance, les descendants du fondateur continuent à intervenir dans ce culte localisé dans un sanctuaire construit par Stèsiléos. À l'époque de la domination athénienne, on ne sait si le culte public persiste ; le sanctuaire de Stèsilos en revanche connaît une fréquentation accrue, mais son public s'est modifié.

Citer ce document / Cite this document :

Durvye Cécile. Aphrodite à Délos : culte privé et public à l'époque hellénistique. In: Revue des Études Grecques, tome 119, Janvier-juin 2006. pp. 83-113.

doi : $10.3406 /$ reg.2006.4647

http://www.persee.fr/web/revues/home/prescript/article/reg_0035-2039_2006_num_119_1_4647 
Cécile DURVYE

\title{
APHRODITE À DÉLOS : CULTE PRIVÉ ET PUBLIC À L'ÉPOQUE HELLÉNISTIQUE
}

\begin{abstract}
RÉSUMÉ. - Cette communication, prononcée le 5 décembre 2005 lors d'une séance de l'Association des Études grecques, a pour objet de proposer une synthèse de nos connaissances sur le culte d'Aphrodite à Délos à l'époque hellénistique. Ce culte revêt trois formes principales. Un culte public donnant lieu à des fêtes, les Aphrodisia, est pratiqué pendant toute la période de l'Indépendance délienne (314-167 av. J.-C.). Parallèlement, un culte présentant de forts caractères familiaux est fondé à la fin du $\mathrm{IV}^{\mathrm{e}}$ siècle par un Délien nommé Stèsiléos; pendant l'Indépendance, les descendants du fondateur continuent à intervenir dans ce culte localisé dans un sanctuaire construit par Stèsiléos. À l'époque de la domination athénienne, on ne sait si le culte public persiste; le sanctuaire de Stèsilos en revanche connaît une fréquentation accrue, mais son public s'est modifié.
\end{abstract}

ABstract. - This paper was presented the $5^{\text {th }}$ December 2005 at the Association des Etudes grecques. Its purpose is to offer a synthesis of our knowledge concerning the cult of Aphrodite at Delos during the Hellenistic times. This cult has three main forms. A public cult including festivals, the Aphrodisia, is practised during all Delos Independence (314-167 BC). Another cult characterized by a strong familial dimension is created at the end of the $4^{\text {th }}$ century by a Delian named Stesileos who founded a sanctuary; during the time of Delos Independence, the founder's descendants maintain the cult. We don't know if the public cult survives in the period of the Athenian domination, but the sanctuary of Stesileos develops and welcomes numerous and quite different worshippers.

Dans ses Recherches sur les cultes de Délos à l'époque hellénistique et à l'époque impériale, Philippe Bruneau a rassemblé les 
documents disponibles sur le culte d'Aphrodite à Délos ${ }^{1}$ pour dresser un tableau de ce culte qui forme un volet de sa présentation générale des cultes pratiqués à Délos. À la lumière des récentes découvertes ${ }^{2}$ et d'une recherche spécifiquement consacrée à Aphrodite, j'essaierai ici d'analyser différentes pratiques cultuelles adressées à Aphrodite à Délos, soit à l'échelle de la cité, soit à une échelle plus réduite.

Un culte public est rendu à la déesse dès le début de l'époque hellénistique ; parallèlement à ce culte officiel apparaît à l'époque de l'Indépendance (c'est-à-dire au début de l'époque hellénistique, puisque Délos recouvre son indépendance en 314) un culte fondé à titre privé par un Délien nommé Stèsiléos. En 167, Rome livre Délos à Athènes et la population délienne est expulsée de l'île ; le culte fondé par Stèsiléos change alors de statut.

\section{Pratique publique}

\section{L'Aphrodite de Thésée}

L'Hymne à Délos de Callimaque, rédigé au début du $\mathrm{III}^{\mathrm{e}}$ siècle, fait coïncider avec le passage de Thésée à Délos l'installation dans l'île d'une statue d'Aphrodite : la tradition littéraire, dont Callimaque est le plus ancien représentant, affirme unanimement que Thésée, après avoir abandonné Ariane à Naxos, s'est arrêté à Délos et y a déposé une statue d'Aphrodite. Le texte de Callimaque est le suivant (Hymne à Délos, v. 307-309) :

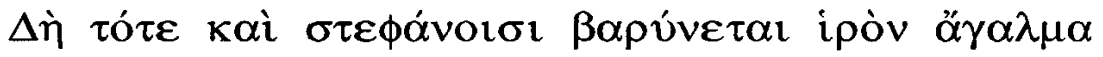

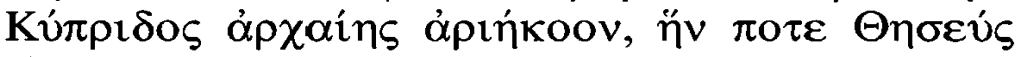

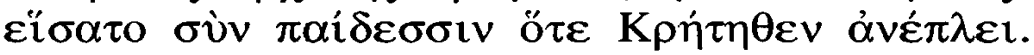

On pourrait le traduire ainsi : «C'est alors que l'on charge de couronnes l'illustre statue sacrée de l'antique Cypris, que déposa Thésée en revenant de Crète avec les jeunes gens ». Le texte apporte deux éléments concernant une célébration d'Aphrodite

\footnotetext{
${ }^{1} \mathrm{Ph}$. Bruneau, Recherches sur les cultes de Délos à l'époque hellénistique et à l'époque impériale, BEFAR 217 (1970), p. 331-348, abrégé désormais en CDH.

${ }^{2}$ L'étude et la fouille de l'Aphrodision, commencées en 1912 par P. Roussel (Délos colonie athénienne, BEFAR 111 [1916, deuxième édition augmentée de compléments bibliographiques 1987], citć désormais $D C A$, p. 240-242) et poursuivies en 1946 par H. Gallet de Santerre et J. Tréheux ( $B C H$ 71-72 [1947-48], p. 411-412) puis en 19571958 par Fr. Salviat $(B C H 82[1958]$, p. 821), ont été reprises en 2005.
} 
qui remonterait aux époques légendaires : il indique la présence à Délos d'un ö $\gamma \alpha \lambda \mu \alpha$ ancien et vénérable venu de Crète et évoque une fête lors de laquelle la statue est couronnée. Les vers qui suivent immédiatement ce passage lient la célébration d'Aphrodite à la géranos, une danse menée pour la première fois par Thésée et ses compagnons autour d'un autel ${ }^{3}$. Malgré l'ambiguïté du texte, il a été clairement démontré que cet autel était celui d'Apollon ${ }^{4}$ et que la géranos n'est pas une manifestation destinée à Aphrodite. Il faut toutefois noter que la célébration des deux rites, couronnement d'Aphrodite et danse de la géranos, est présentée par Callimaque comme concomitante et de même origine; le fait n'est pas négligeable, la danse de la géranos étant l'un des rituels déliens les plus anciens et les plus sacrés.

La statue d'Aphrodite apportée de Crète par Thésée est évoquée par deux autres témoignages littéraires plus tardifs, un passage de Plutarque 5 et un autre de Pausanias. Le texte de Plutarque présente une analogie frappante avec le passage de Callimaque.

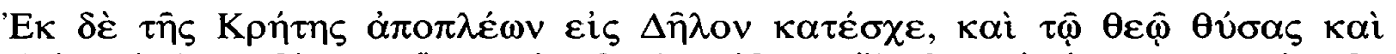

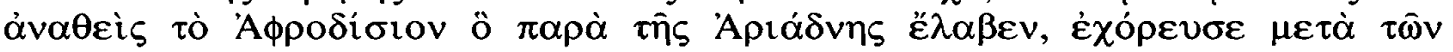

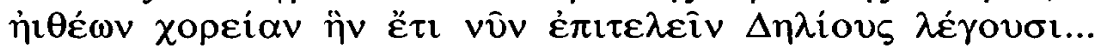

"Revenant de Crète, (Thésée) aborda à Délos, et, après avoir sacrifié au dieu et consacré la statue d'Aphrodite qu'il avait reçue d'Ariane, il exécuta avec les jeunes gens un chœur de danse que l'on dit être encore en usage aujourd'hui chez les Déliens...»

La figure d'Ariane n'apparaissait pas chez Callimaque ${ }^{6}$ et Plutarque ne mentionne pas le couronnement de la statue. À ces deux éléments près, la tradition est visiblement la même que dans le texte précédent : on y retrouve le retour de Crète, le dépôt de la statue et la danse. La similitude entre les deux textes est très grande, aussi bien par l'ordre des faits rapportés (dépôt de la statue, puis danse) que par les éléments en présence (mention de la Crète, du voyage par mer, présence des compagnons de Thésée). De légères différences de vocabulaire sont notables : là

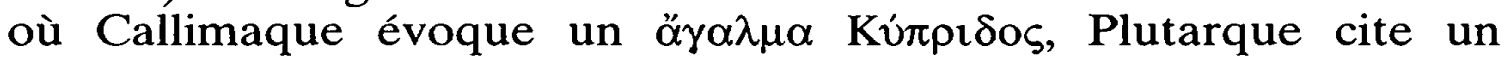

3 Sur la géranos, voir entre autres Ph. Bruneau, $C D H$, p. 29-32 ; «Deliaca (VII), 54 : Toujours la géranos », $B C H 112$ (1988), p. 575-577.

4 Ph. Bruneau, $C D H$, p. 23.

5 Vie de Thésée, 21, 1.

6 Sur les rapports entre Thésée, Ariane et Aphrodite, cf. V. Pirenne-Delforge, L'Aphrodite grecque, Kernos Suppl. 4 (1994), p. 166 et 349-350. Sur une possible assimilation d'Ariane et Aphrodite à Délos, cf. H. Gallet de Santerre, Délos primitive et archaïque, BEFAR 192 (1958), p. 153-154. 


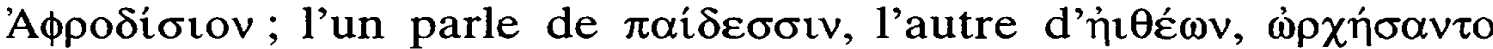

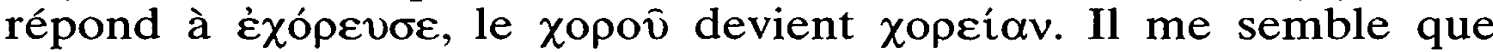
ces différences, sur une tradition si proche, peuvent n'être pas le fruit du hasard : je considérerais volontiers que Plutarque cherche par ces variations systématiques à se démarquer de Callimaque.

Le troisième texte citant la statue crétoise d'Aphrodite est un passage de Pausanias qui, dans son livre sur la Béotie, dresse une liste des œuvres de Dédale ${ }^{7}$.

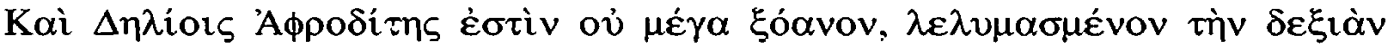

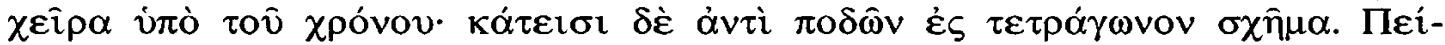

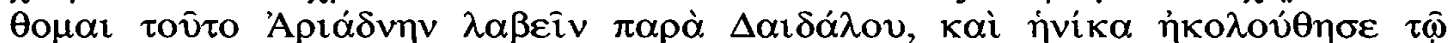

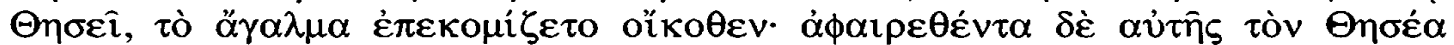

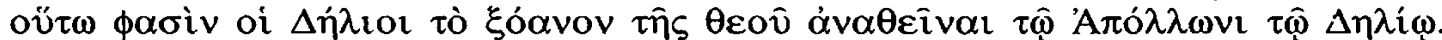

«Il y a aussi chez les Déliens un petit xoanon d'Aphrodite, abîmé à la main droite par le temps; il s'achève, à la place de jambes, en forme de parallélépipède ${ }^{8}$. Je crois que c'est celui-ci qu'Ariane reçut de Dédale, et que lorsqu'elle accompagna Thésée, elle emporta la statue de chez elle. Lorsque Thésée l'abandonna, selon les Déliens, il consacra le xoanon de la déesse à Apollon Délien ».

La tradition rapportée ici diffère un peu de la précédente : le thème de la consécration de la statue est isolé et développé. Deux éléments nouveaux sont apportés. Le premier est une description de la statue : le terme xoanon désigne chez Pausanias une statue ancienne, probablement en bois ${ }^{9}$, et concorde donc avec la formule employée par Callimaque. Il s'agit apparemment d'une représentation partiellement figurative puisqu'elle a des mains, mais se termine en pilier $^{10}$. Le deuxième élément nouveau est l'attribution de la statue à Dédale.

La seule certitude qu'apportent les témoignages littéraires est celle de la présence dans le sanctuaire d'Apollon, entre l'époque de Callimaque et celle de Pausanias, d'une statue d'Aphrodite

7 IX 40, 3-4. Sur Pausanias et Délos, voir A. Jacquemin, «Pausanias à Délos ou un chapitre recomposé du livre imaginé des Kykladika », Ktema 25 (2000), p. 19-36.

8 C'est-à-dire en fût hermaïque : cf. Ph. Bruneau, $C D H$, p. 334 n. 4.

9 Toutes les statues de Dédales, dans ce passage, sont appelées $\xi o ́ \alpha v \alpha$ par Pausanias, qui utilise très fréquemment le terme pour des statues d'Aphrodite. Sur les xoana et leur matériau, voir entre autres W. K. Pritchett, Pausanias Periegetes (1998), p. 204294 ; pour une analyse diachronique de l'emploi du mot, voir A. A. Donohue, Xoana and the Origin of Greek Sculpture (1988).

10 Sur les statues-piliers féminines, voir R. Lullies, Die Typen der griechischen Hermen, 1931 , p. 55, et A. Jacquemin, «Une statue féminine thasienne», $B C H 108$ (1984), p. $447-456$. 
considérée comme ancienne et d'origine crétoise. Le passage de Thésée à Délos est traditionnellement considéré comme fondateur d'un certain nombre de rites spécifiques, en particulier la danse de la géranos, dont l'étrangeté semble attester l'archaïsme, même si nous n'en avons que des témoignages récents. La statue d'Aphrodite est liée à ces rites puisque dans deux des témoignages qui la mentionnent, elle apparaît juste avant la danse de la géranos; mais elle n'en est qu'un élément très secondaire, et n'est même pas citée dans la plupart des textes qui parlent de la géranos, de l'autel de cornes, des jeux et des concours institués par Thésée ${ }^{11}$. Le rôle de la statue n'est pas assuré. Le don du xoanon d'Aphrodite n'apparaît pas nécessairement comme la fondation d'un nouveau culte, mais simplement comme une offrande à Apollon : Plutarque et Pausanias emploient le verbe $\dot{\alpha} v \alpha \theta \varepsilon i v \alpha \imath$, Callimaque le terme plus neutre de $\varepsilon i ̈ \sigma \alpha \tau o$. L'hypothèse d'un culte lié à cette statue ne repose que sur le texte de Callimaque, qui en rapporte le couronnement annuel. Aucun des textes ne donne d'indication sur le contexte matériel de l'installation de la statue à Délos; il semble toutefois probable qu'elle ait été déposée dans le sanctuaire d'Apollon, puisqu'elle lui est consacrée.

\section{Le culte public}

En dehors du domaine légendaire dont relèvent ces textes, nous n'avons aucune mention littéraire de la statue d'Aphrodite. Toutefois la présence de la déesse dans le sanctuaire d'Apollon est attestée par un certain nombre d'inscriptions, en particulier un type de documents qui ne laisse aucun doute quant à la célébration d'un culte public à Aphrodite pendant la période de l'Indépendance : les inscriptions relatives aux Aphrodisia. L'importance accordée à cette Aphrodite officielle peut être mesurée au nombre et à la nature des offrandes qui lui sont dédiées ${ }^{12}$.

Les comptes des magistrats responsables de l'administration du sanctuaire d'Apollon pendant la période de l'Indépendance, les hiéropes, mentionnent des dépenses relatives à la fête des Aphro-

11 Ces textes ont été rassemblés et commentés par Ph. Bruneau, CDH, p. 19-35.

12 Je ne développerai pas ici le rôle joué par Aphrodite associée à Hermès en tant que protectrice des magistrats : il ne semble pas donner lieu à Délos à des célébrations spécifiques. Sur ce point, on pourra consulter Ph. Bruneau, CDH, p. 344-345; sur le même phénomène à Thasos et dans le reste de la Grèce, Fr. Salviat et Fr. Croissant, «Aphrodite gardienne des magistrats : gynéconomes de Thasos et polémarques de Thèbes », BCH 90 (1960), p. 460-471. 
disia. La célébration de ces fêtes est ainsi attestée régulièrement entre 302 et la fin de la période de l'Indépendance, les dernières inscriptions étant datées entre 175 et 167 ; il se peut qu'elles aient continué par la suite, mais nous n'en avons pas gardé trace. La fête, qui semble annuelle, se déroule au mois Hécatombaion (juilletaoût), puisque c'est sous la rubrique des dépenses de ce mois que sont enregistrés les frais qu'elle occasionne. La nature des dépenses effectuées donne quelques éléments d'information sur le déroulement de la fête. Les comptes mentionnent plusieurs achats : Aфро-

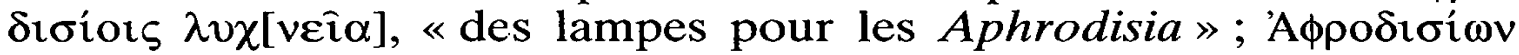
$\tau \bar{\omega} \iota$ $\chi 0 \rho \hat{\imath} \iota \lambda \alpha \mu \pi \alpha ́ \delta \varepsilon \zeta$, des torches pour le chœur des Aphrodisia»;

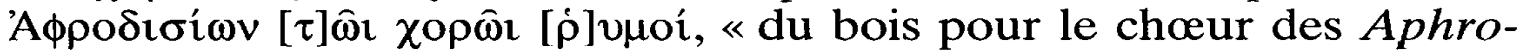

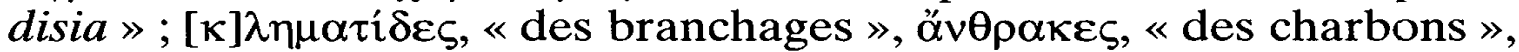
et des $\chi 0 \rho \varepsilon i \hat{\alpha}$ qui semblent être des fournitures destinées au chœur. Le matériel acquis pour les Aphrodisia montre qu'il s'agit de fêtes

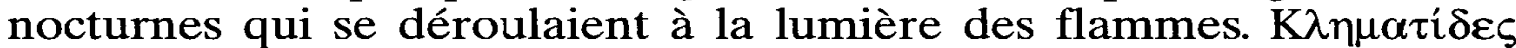
et $[\dot{\rho}] v \mu o i ́$, selon Ph. Bruneau, sont destinés à allumer les $\lambda \alpha \mu \pi \alpha \dot{\alpha} \delta \varepsilon \zeta$

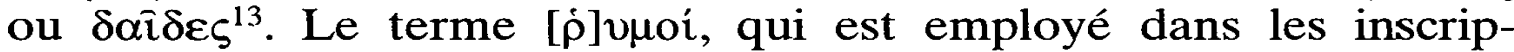
tions déliennes pour désigner toutes sortes d'objets en bois, semble renvoyer ici à un «type de bois de chauffage acheté en rondins ${ }^{14}$; il s'agit peut-être simplement de bûches utilisées, avec les branchages et les charbons, pour entretenir le foyer de l'autel ou quelques braseros. Le chœur, dans la mesure où il est mentionné sans plus de précision dans les comptes, doit être le chœur le plus couramment attesté à Délos, celui des Déliades. Callimaque en parle comme d'un chœur de danseuses (Hymne à Délos, v. 306); sous l'Indépendance, ce chœur se produit lors de plusieurs fêtes (Apollonia, Artémisia, Aphrodisia, Antigoneia, Ptolémaia, etc.). Les comptes conservés ne portent trace ni de sacrifice ni de banquet, et le lieu de célébration nous est inconnu.

Un culte officiel d'Aphrodite, financé par les hiéropes et comportant des fêtes célébrées de nuit par un chœur est donc pratiqué à Délos pendant toute la période de l'Indépendance.

Outre les comptes, les offrandes consacrées à Aphrodite dans le sanctuaire d'Apollon sont pour nous un moyen de mesurer le rôle de la déesse dans le sanctuaire officiel de la cité. La nature de ces offrandes nous est connue par les inventaires. Les textes conservés datent du début de l'Indépendance jusque vers 140 ;

$13 \mathrm{CDH}$, p. 37.

14 Cl. Prêtre (éd.), Nouveau choix d'inscriptions de Délos. Lois, comptes et inventaires, Études épigraphiques 4 (2002), p. 274. 
ceux qui mentionnent des objets consacrés à Aphrodite déposés dans le sanctuaire d'Apollon sont répartis entre le début du $\mathrm{III}^{\mathrm{e}}$ siècle et la fin de la période athénienne. Les offrandes à Aphrodite sont à vrai dire très peu nombreuses. On trouve dans les inventaires de l'Artémision (le sanctuaire d'Artémis est inclus dans celui d'Apollon) : une $\kappa u ́ \lambda t \xi$ d'or, offerte à Aphrodite par Ptolémée fils de Lagos, qui est mentionnée pour la première fois au milieu du III $^{\mathrm{e}}$ siècle, mais dont le dépôt est nécessairement antérieur à 283 , date de la mort de Ptolémée ${ }^{15}$; une phiale d'argent à reliefs, offrande d'Échénikè ${ }^{16}$; une $\kappa u ́ \lambda \iota \xi$ en or, offrande d'Échénikè, d'un poids de 50 drachmes ${ }^{17}$. Une autre offrande à Aphrodite est conservée dans le sanctuaire d'Eileithyie ${ }^{17 a}$ : il s'agit

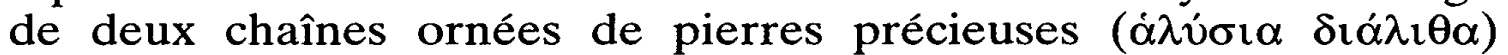
consacrées à Aphrodite par un dénommé Aristonicos, attestées en 276 et $274^{18}$. Ces offrandes dédiées à Aphrodite dans le sanctuaire d'Apollon sont certes précieuses, mais rares; l'une vient d'un étranger, Ptolémée, les autres de Déliens. Les dévots du culte officiel d'Aphrodite semblent donc peu nombreux.

Le lieu de conservation de ces offrandes appelle une remarque concernant le sanctuaire officiel d'Aphrodite; à part l'offrande d'Aristonicos, elles sont déposées dans l'Artémision. Sans être probant, le fait incite à considérer la possibilité qu'il n'ait pas existé d'Aphrodision susceptible de contenir des offrandes dans le sanctuaire d'Apollon; de fait, aucun vestige d'un sanctuaire d'Aphrodite n'a été identifié ni dans le sanctuaire d'Apollon ni dans les environs immédiats ${ }^{19}$.

Pourtant, les comptes des hiéropes mentionnent deux édifices différents désignés sous le nom d'Aphrodision : l'un se situe en dehors du sanctuaire d'Apollon - il s'agit de l'Aphrodision de Stèsiléos, dont nous traiterons plus bas -, mais l'autre se trouve apparemment dans le sanctuaire même. Tous deux sont cités dans un même compte de l'année 246 qui indique, dans deux passages

15 IG XI, 2, 161, B, 1. 26-27. Ph. Bruneau propose de la dater de 308 (CDH, p. 516).

$16 I G \mathrm{XI}, 2,287, B, 1.75$. Échénikè est la fille du Stèsiléos qui fonda un Aphrodision dont nous traiterons plus bas.

17 IG XI, 2, 287, B, 1. 32 .

17 a C'est-à-dire probablement dans le sanctuaire d'Apollon : voir Ph. Bruneau, $C D H$, p. 213.

18 IG XI, 2, 164, B, 1. 5 et $199, B, 1.67$.

19 Sur la tentative d'identification de l'Aphrodision avec le sanctuaire dit « du bastion » $\left(\mathrm{n}^{\circ} 72 \mathrm{du}\right.$ Guide de Délos, Sites et monuments 1, 4 éd. 2005 , abrégé désormais en $G D$ ), cf. Ph. Bruneau, $C D H$, p. 338-339. Sur la tentative d'identification de l'Aphrodision avec le «temple $\mathrm{G} »(G D 40)$, voir Ph. Bruneau, $C D H$, p. 334. 
indépendants, les dépenses effectuées d'une part pour la répara-

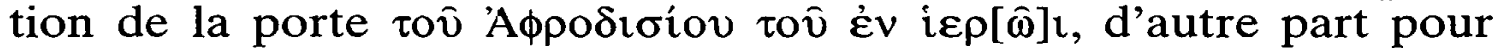

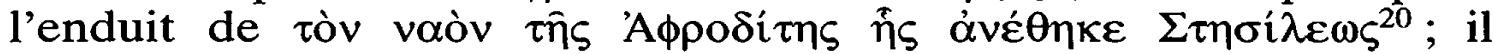
semble bien que les deux bâtiments soient distincts et il faut donc

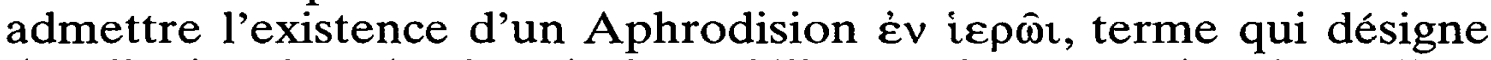
d'ordinaire dans les inscriptions déliennes le sanctuaire d'Apollon.

Puisque l'on reconnaît l'existence de deux bâtiments distincts, il faut répartir les inscriptions évoquant un Aphrodision entre les deux sanctuaires d'Aphrodite, l'Aphrodision du sanctuaire d'Apollon et le sanctuaire «de l'Aphrodite qu'a consacrée Stèsiléos». Si les inscriptions décrivaient des édifices de nature clairement différente, cette répartition serait aisée, mais les termes employés par les magistrats sont très généraux. Beaucoup de passages des comptes et des inventaires citant un Aphrodision peuvent se rapporter au sanctuaire de Stèsiléos tel que nous le connaissons par les vestiges qui en subsistent; rares sont les textes employant des termes qui ne semblent pas pouvoir désigner des édifices appartenant à ce sanctuaire. Il est toutefois question dans une inscription d'une réparation faite aux portes du vaï $\sigma \kappa o s$ d'Aphrodite ${ }^{21}$; or la plupart des inscriptions relatives à un Aphrodision parlent de vaós d'Aphrodite, y compris celle que nous venons de citer et qui se réfère explicitement à l'Aphrodision de Stèsiléos. Ph. Bruneau estime que le terme voḯoко s ne peut s'appliquer à l'Aphrodision de Stèsiléos et doit se rapporter à l'Aphrodision év i déliens utilisent d'ordinaire vơḯós pour désigner des offrandes qui semblent avoir la forme d'« un simple ex-voto en bois ou en pierre, comme ceux qui ont été retrouvés, où la figure de la divinité se dégage d'un encadrement architectural à frontons sur pilastres ${ }^{23}$. Si l'Aphrodision év i $\varepsilon \rho \hat{\omega} \iota$ contient la statue de Thésée, il peut s'agir d'un équivalent de grandes dimensions de ces objets. Mais le temple construit par Stèsiléos étant de petite taille, il n'est pas invraisemblable qu'un magistrat ait pu employer le terme

20 ID 290 , l. 84 et 151 .

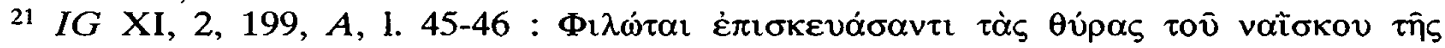

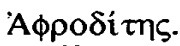

22 Ph. Bruneau, $C D H$, p. 333.

23 M.-Chr. Hellmann, Recherches sur le vocabulaire de l'architecture grecque d'après les inscriptions de Délos, BEFAR 278 (1992), p. 271. Une comparaison des occurrences du terme dans les inscriptions déliennes inciterait à le définir encore plus vaguement comme un «présentoir» pouvant contenir ou supporter divers objets ( $I G X I, 2,161$, $B$, 1. $79 ; 203, B, 1.85 ; 287, B, 1.17 ; 320, B, 1.75)$. En dehors du passage qui concerne l'Aphrodision, le mot n'est employé qu'une fois pour désigner un édifice : dans l'oikos proche de l'Ecclèsiastèrion se trouve le modèle réduit d'un väióó̧ de bois (ID 1417, $A$, col. I, 1. 32). 
vaïokos comme un simple diminutif de vaós. Mentionnons aussi, pour mémoire, une inscription évoquant une réparation de la table du $\sigma \tau \omega \ddot{i} \delta i ́ \omega \imath$ de l'Aphrodision ${ }^{24}$; aucun portique n'est visible aujourd'hui dans le sanctuaire de Stèsiléos, mais l'argument n'a en aucune façon valeur de preuve et il faudra se contenter de savoir que l'un des deux édifices a possédé un portique.

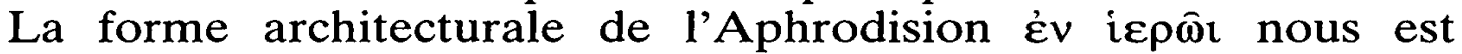
donc presque totalement inconnue, la seule inscription qui s'y rapporte de façon certaine nous apprenant uniquement qu'il était muni de portes. Dans la mesure où les offrandes à Aphrodite sont conservées dans l'Artémision et non dans l'Aphrodision غ̇v

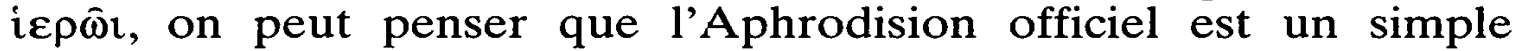
édicule muni de portes et contenant peut-être la statue d'Aphrodite attribuée à Dédale ${ }^{25}$. On ne peut exclure l'existence d'un véritable temple dédié à Aphrodite; mais le dépôt des offrandes à Aphrodite dans le sanctuaire d'Artémis fait pencher en faveur

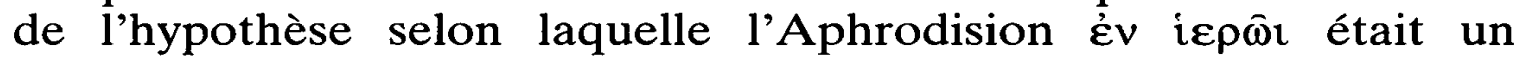
édifice certes pourvu de portes, mais inapte à recevoir des offrandes, c'est-à-dire un ouvrage de l'ordre de la chapelle.

\section{Synthèse}

Il existe assurément un culte officiel d'Aphrodite à Délos à l'époque hellénistique, puisque des fêtes sont financées par le sanctuaire d'Apollon; mais nous en savons très peu quant à son organisation matérielle. Il n'a laissé aucun vestige identifié, ni bâtiment, ni offrandes, ni statuaire, ni inscriptions votives en place; la seule manifestation certaine du culte est la fête des Aphrodisia, que l'on peut éventuellement mettre en relation avec le couronnement de la statue mentionné par Callimaque. Les textes évoquent une statue ancienne encore en place à l'époque hellénistique, dont on peut supposer qu'elle était installée dans le grand sanctuaire, dans une chapelle munie de portes. Malgré la très probable absence d'édifice indépendant et la rareté des offrandes dans le sanctuaire officiel, l'existence d'une fête portant le nom d'Aphrodite montre que la déesse faisait partie du panthéon délien, sans statut commun bien sûr avec la triade apollinienne ou Héra, mais sur le même plan que tout un groupe de dieux secondaires comme Dionysos, Eileithyie, Asclépios, etc.

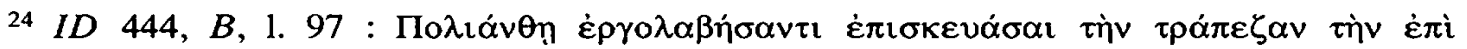

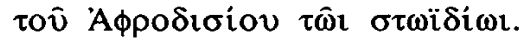

25 Sur la présence possible d'une offrande dans cet édicule, voir plus bas p. 99. 


\section{Pratique familiale : L'APhrodite de Stèsiléos}

Si nous avons assez peu de renseignements sur le culte officiel, nous connaissons en revanche très bien un culte d'Aphrodite qui relève d'une pratique assurément familiale et sans doute privée. Ce culte, fondé par un Délien, nommé Stèsiléos, est entretenu par sa famille pendant toute la période de l'Indépendance.

Le sanctuaire d'Aphrodite fondé par Stèsiléos est situé à l'écart du sanctuaire d'Apollon (Fig. 1), à la limite du plus ancien des quartiers d'habitation déliens, le Quartier du théâtre ${ }^{26}$. Il est composé d'un tcmplc dc pctites dimensions dont l'entrée est flanquée par deux bases inscrites, d'un autel à frontons et de plusieurs constructions faites chacune d'une pièce ouvrant sur une cour (Fig. 2). La documentation concernant l'Aphrodision de Stèsiléos est exceptionnelle, même à Délos, car l'identification assurée du sanctuaire permet de mettre en relation des vestiges bien conservés avec des inscriptions de diverse nature, et d'obtenir par là une image particulièrement nette de l'histoire du sanctuaire pendant toute sa période de fonctionnement entre la fin du $\mathrm{IV}^{\mathrm{e}}$ et le début du $\mathrm{I}^{\text {er }}$ siècle. Dans l'étude de cet Aphrodision, une articulation chronologique s'impose : en expulsant les Déliens en 167 et en s'installant à leur place sur l'île, les Athéniens provoquent une brusque rupture dans l'occupation des lieux et le statut de l'Aphrodision de Stèsiléos s'en trouve modifié. Nous diviserons donc la présentation du sanctuaire en deux phases en étudiant d'abord l'Aphrodision de l'Indépendance, puis l'Aphrodision de l'époque athénienne, et en exposant pour l'un comme pour l'autre ce qu'inscriptions et vestiges permettent de savoir à la fois du sanctuaire et de ses éléments constituants, des modalités du culte et du statut du sanctuaire et du culte.

\section{Le sanctuaire sous l'Indépendance}

Tout au début de l'Indépendance, un notable délien nommé Stèsiléos consacre à Aphrodite un petit sanctuaire composé d'un temple, d'un autel et d'un bâtiment annexe (Fig. 3). Devant le temple, deux bases de marbre supportaient des statues de bronze. L'ensemble de ces constructions forme un sanctuaire dont les limites sont mal connues.

${ }^{26}$ Sur cette chronologie, cf. Ph. Bruneau, «Contribution à l'histoire urbaine de Délos », BCH 92 (1968), p. 639. 
L'élément principal du sanctuaire, la statue de culte, a aujourd'hui disparu. Son existence est certifiée par un compte qui mentionne explicitement une statue d'Aphrodite comme «consacrée par Stèsiléos » : en 246, les hiéropes paient un ouvrier chargé

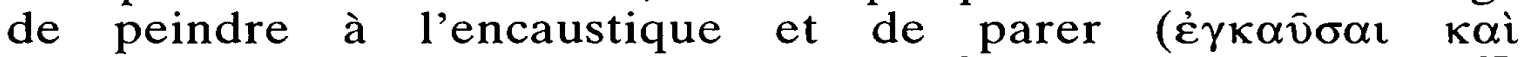

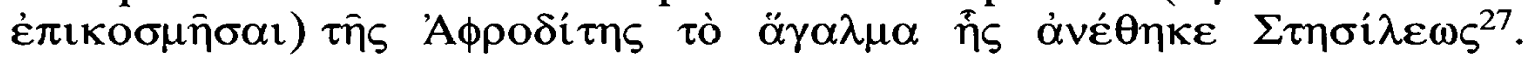
Cette statue est décrite dans des inventaires de l'époque athé-

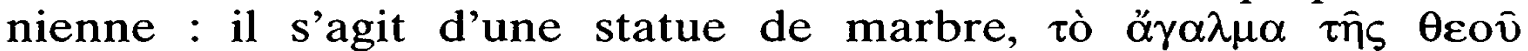

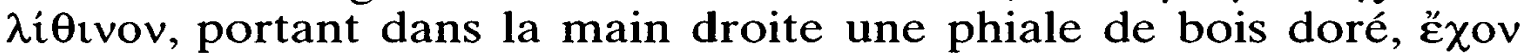

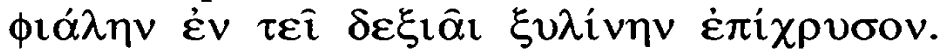

La première attestation d'une statue d'Aphrodite dans les comptes date de 304 : c'est le paiement d'un artisan nommé Nicôn

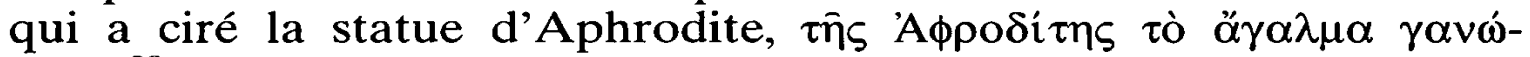
$\sigma \alpha \nu \tau \imath^{28}$. Par la suite, les comptes portent de nombreux paiements pour la $\gamma \alpha v \bar{\omega} \sigma \iota \varsigma$, l'é $\pi$ เ tique) d'une statue d'Aphrodite; les mêmes comptes mentionnent souvent l'achat de $\pi \varepsilon \tau$ đó $\lambda \alpha$ (feuilles d'or). Ces divers travaux opérés sur la statue sont généralement payés en Hécatombaion, qui est le mois de la célébration des Aphrodisia officiels. La difficulté est de savoir si tous les comptes se rapportent à la statue de Stèsiléos, ou si les deux statues, celle de Thésée et celle de Stèsiléos, font l'objet d'un entretien annuel. Voyons donc quels arguments parlent en faveur de ces hypothèses, et comment ils peuvent être réfutés.

1) Nous n'avons aucune attestation archéologique de la statue de Thésée, qui n'apparaît dans aucun des inventaires du sanctuaire d'Apollon et n'a laissé aucun vestige identifié. Mais l'absence de vestige n'a rien de concluant, et l'ignorance totale de cette statue par les inventaires n'est pas un cas unique.

2) Dans l'une des assez nombreuses occurrences où l'achat de $\pi \varepsilon \tau \dot{\lambda} \lambda \alpha$ est associé au nettoyage et à la parure de la statue d'Aphrodite, celle-ci est explicitement désignée comme celle de Stèsiléos ${ }^{29}$. $J$. Tréheux en conclut que toutes les inscriptions où il est fait mention de feuilles d'or se rapportent à la statue consacrée par Stèsiléos. Le raisonnement aurait plus de poids si les $\pi \varepsilon \tau \alpha ́ \lambda \alpha$, dans

27 ID 290, 1. 51. Sur les différents termes techniques se rapportant à l'entretien de la statue, cf. J. Marcadé, Au Musée de Délos, BEFAR 215 (1969), p. 98-102. Nous reviendrons plus bas sur le financement par les hiéropes de l'entretien du sanctuaire de Stèsiléos.

${ }^{28} I G X I, 2,144, B, 1.5$.

29 ID 290, 1. 153. L’argument, développé par J. Tréheux dans ses Études critiques sur les inventaires de l'Indépendance délienne (thèse manuscrite, Paris, 1959, p. 442443), est repris par Ph. Bruneau, CDH. p. 336. 
l'inscription ID 290, n'étaient pas restitués. Il est possible que le xoanon de Thésée ait été doré ${ }^{30}$. De plus, la précision apportée par le rédacteur du compte mentionnant qu'il s'agit de la statue consacrée par Stèsiléos pourrait donner lieu à l'interprétation inverse : si cette précision est donnée, c'est que la statue qui reçoit d'ordinaire ce traitement n'est pas celle de Stèsiléos, mais celle de Thésée... On pourrait aller jusqu'à supposer que les mentions de la statue d'Aphrodite dans les comptes ne se rapportent à la statue de Stèsiléos que quand celle-ci est explicitement mentionnée ; mais l'interprétation paraît hasardeuse, et les arguments avancés en laveur de l'hypothèse contraire (absence de vestige, absence de mention spécifique dans les inventaires, restitution de $\pi \varepsilon \tau \alpha$ $\lambda \alpha \alpha$ liés à l'Aphrodite de Stèsiléos) me semblent convaincants, sinon probants. Je tends donc à penser que toutes les mentions de la statue d'Aphrodite dans les comptes et les inventaires concernent la statue consacrée par Stèsiléos et que l'on peut considérer sa plus ancienne attestation comme le compte de 304 cité plus haut. J'avancerai toutefois cette hypothèse avec moins de certitude que ne le font $\mathrm{J}$. Tréheux et après lui $\mathrm{Ph}$. Bruneau.

La statue reposait sur une base dont la fondation est encore en place dans le temple (Fig. 4); sur cette fondation ont été remontés deux blocs de la première assise, sur lesquels on voit les traces de scellements en double $T$ qui ne permettent pas de dater le monument, mais dont nous seront amenés à reparler plus bas.

Le temple est une construction de petites dimensions $(4,13 \mathrm{~m}$ de façade sur 7,04 m de profondeur). Il n'est pas question d'en faire une description complète qui n'aurait guère sa place ici ; notons néanmoins quelques particularités qui en font un monument original. L'Aphrodision, comme l'Héraion archaïque de Délos, est orienté vers le Sud. Ses murs de marbre blanc présentent un appareil original de blocs polygonaux à joints obliques et lits généralement parallèles (Fig. 5) : c'est là un appareil rare, dont les seuls exemples à Délos datent de l'Indépendance et qui n'a de parallèles que dans deux ou trois autres monuments de cette période $^{31}$. Le plan de l'édifice est inhabituel puisqu'il se présente

\footnotetext{
30 Pausanias mentionne plusieurs xoana dorés (II 2,$6 ;$ VI 24, 6; 25, 4 ; VII 26, $4 ;$ IX 4, 1).

31 Ces parallèles sont l'habillage de marbre du Réservoir de l'Inopos (GD 97), dans une moindre mesure le mur de soutènement du théâtre (GD 114), dont les blocs présentent des bossages, et la partie basse des murs de la Salle hypostyle (GD 50) dont les blocs sont beaucoup plus homogènes en taille que ceux de l'Aphrodision et présentent moins de décrochements.
} 
comme un corps rectangulaire aptère. Ce corps est divisé en deux pièces, une cella de $2,64 \mathrm{~m}$ de profondeur et un pronaos légèrement plus grand $(2,92 \mathrm{~m}$ de profondeur); ce rapport de proportions est tout à fait exceptionnel. Une mosaïque d'éclats de marbre couvre le sol du temple ${ }^{32}$. Dans le pronaos, deux pieds de bancs en marbre sont pris dans la mosaïque et les fondations de trois autres sont visibles : deux bancs longeaient les murs Est et Ouest de la pièce. Devant l'emplacement des bancs, la mosaïque présente une usure témoignant d'un usage intensif ou prolongé qui indique que le temple a connu une fréquentation inhabituelle.

Le premier compte où l'Aphrodision de Stèsiléos est identifié comme tel date de 246 et a été cité plus haut : il mentionne à

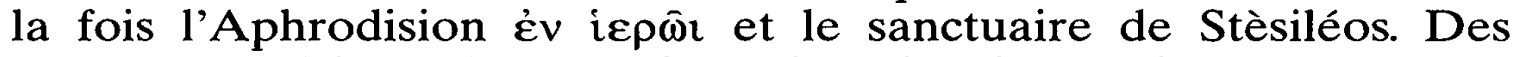
comptes antérieurs à cette date, dont le plus ancien remonte au début $\mathrm{du}^{\mathrm{III}} \mathrm{e}^{\mathrm{e}}$ siècle, mentionnent un Aphrodision qui est très probablement, mais non certainement, celui de Stèsiléos. Quant au premier inventaire où l'on identifie assurément l'Aphrodision de Stèsiléos, il date de l'époque athénienne, un peu avant $156^{33}$.

Un argument incite toutefois à faire remonter la construction du temple à la fin du IV siècle : c'est la présence devant le temple, de part et d'autre de la porte, de deux bases à trois degrés de marbre destinées à des statues de bronze disparues. Chacune de ces bases porte une dédicace : sur la base Est est

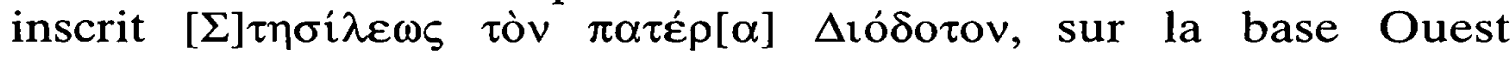

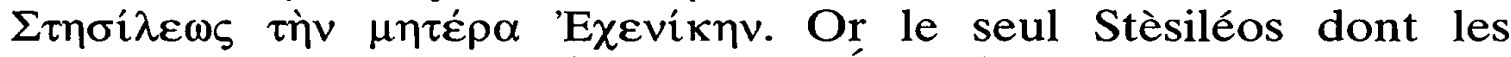
parents se nomment Diodotos et Échénikè est précisément le fondateur du sanctuaire ${ }^{34}$, connu par ailleurs pour avoir été archonte en $305^{35}$; la première attestation de la statue datant de 304 , il semble probable que la statue, le temple et les bases sont contemporains et datent de la fin du IV siècle.

Les comptes des hiéropes attestent un entretien constant du temple pendant la période indépendante : entre 274 et 169 , de nombreuses inscriptions mentionnent des badigeonnages à la poix des boiseries, des achats de clous, des stucages (les parements intérieurs des murs du temple portent encore des traces de stuc) et des réparations de portes. Même s'il est difficile de répartir

32 Cette mosaïque est décrite par Ph. Bruneau dans Les Mosaïques, Exploration archéologique de Délos XXIX (1972), p. 19-22.

${ }^{33}$ ID $1412, a, 1.28-35$.

${ }^{34} \mathrm{Cl}$. Vial, dans son étude sur Délos indépendante, a établi l'arbre généalogique de la famille de Stèsiléos (Délos indépendante, BCH Suppl. X [1984], p. 75).

35 $I G X I, 2,1067, b, 1.5$. 
ces comptes entre l'Aphrodision de Stèsiléos et l'Aphrodision غ́v

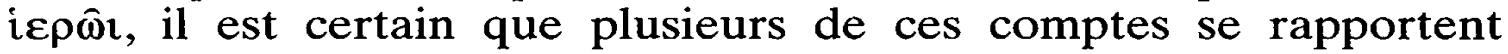
au sanctuaire consacré par Stèsiléos.

Devant le temple se dressait un autel à frontons typiquement hellénistique, tourné vers l'Est (Fig. 6). Sa plinthe moulurée est restée en place; des fragments de la plinthe de couronnement et des deux frontons ont été retrouvés aux alentours, mais ses orthostates ont disparu avec sa plaque de couverture. La contemporanéité de l'autel et du temple est assurée par les traces de scellements en double $\mathrm{T}$ encore visibles sur la plinthe en place, tout à fait identiques à ceux que présente la base de la statue de culte. Cet autel a un parallèle très proche à l'Héraion, où un autel à fronton de dimensions et de forme équivalentes, également orienté à l'Est, a été installé à l'époque hellénistique ${ }^{36}$.

À l'Est du temple d'Aphrodite et aligné sur lui se trouve un édifice à une seule pièce. Il est construit dans un appareil très soigné alternant des blocs de marbre piquetés et des pilettes de gneiss (Fig. 7). L'ensemble évoque l'appareil cycladique courant à Délos dès le $\mathrm{VII}^{\mathrm{e}}$ siècle ; mais celui-ci est d'ordinaire composé de blocs de granit remplacés ici par du marbre. La modification régularise considérablement l'appareil, mais change aussi profondément sa signification. À l'époque archaïque, c'est l'irrégularité des blocs de granit employés qui rend nécessaire l'usage des pilettes; dans le cas de l'Aphrodision, l'emploi de pilettes est un effet de style que le travail aisé du marbre rend superfétatoire ${ }^{37}$.

L'oikos $^{38}$ semble dater de la même période que le temple : non seulement il a exactement la même orientation, mais les blocs de marbre employés pour sa construction ont à peu près les mêmes dimensions moyennes que ceux du temple, et si les appareils du temple et de l'oikos diffèrent, ils sont tous deux extrêmement soignés. Il faudra attendre la fouille de l'oikos pour dater sa construction si faire se peut ; sa contemporanéité avec le temple est probable, mais il n'y en a pour l'instant pas de preuve décisive.

36 Cf. A. Plassart, Les sanctuaires et les cultes du mont Cynthe, EAD XI (1928), p. 206-210. Sur les autels d'Aphrodite et d'Héra à Délos, voir A. Ohnesorg, Ionische Altäre : Formen und Varianten einer Architekturgattung aus Insel- und Ostionien, Archäologische Forschungen 21 (2005), p. 93-95.

37 Il se peut que l'emploj de pilettes soit dicté par un souci d'économie; le soin avec lequel l'assemblage est réalisé me semble toutefois relever de l'effet de style.

38 J'emploie ici le terme oikos qui figure dans les inscriptions (voir ci-dessous p. 104). J'ai numéroté les dépendances du sanctuaire d'Ouest en Est et les désigne désormais comme oikos 1 , oikos 2, etc. 
La destination de cette salle d'une cinquantaine de mètres carrés est mal connue. Son inventeur, P. Roussel, la considérait comme une "sacristie ${ }^{39}$. Les oikoi servent d'ordinaire de lieux de stockage ou de salles de banquet; la porte étant ici centrée, les critères définis par G. Roux ${ }^{40}$ inciteraient à y voir plutôt une réserve, mais les deux usages ne me paraissent pas exclusifs. Il faut espérer que la fouille du bâtiment permettra d'identifier son rôle dans le sanctuaire.

Il n'y a dans les comptes des hiéropes qu'un seul passage que l'on puisse rapporter à cet édifice. En 250, quatre oboles sont

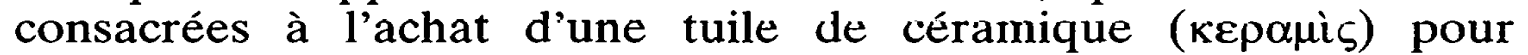
l'Aphrodision $^{41}$; or il semble que le temple ait été au moins partiellement couvert en marbre, puisqu'une grande plaque de marbre à acrotères est conservée à proximité. Cette tuile de terre cuite peut donc être destinée à une dépendance du temple, peutêtre à celle que nous venons de décrire.

Temple, bases, autel et oikos forment un ensemble architectural cohérent à tous points de vue : dans l'espace, puisque les constructions sont regroupées et que leurs orientations sont identiques, à l'exception de celle de l'autel; chronologiquement, puisque l'ensemble paraît contemporain; qualitativement enfin puisque toutes ces constructions de marbre sont très soignées. Le sanctuaire est de dimensions modestes, mais de belle qualité; il a apparemment été offert dans son ensemble par Stèsiléos dans les dernières années $\mathrm{du} \mathrm{IV}^{\mathrm{e}}$ siècle et a été régulièrement entretenu par les hiéropes pendant l'Indépendance.

\section{Le culte}

La forme du culte pratiqué dans ce sanctuaire est partiellement connue grâce aux inscriptions. Celles-ci mentionnent deux fêtes fondées par des membres de la famille de Stèsiléos, l'une par Stèsiléos lui-même en 302, l'autre par sa fille Échénikè en $250^{42}$. Ces fêtes sont des fêtes de fondation, c'est-à-dire que leur fondateur dédie une somme qui, gérée par les hiéropes, produit chaque année un revenu du dixième consacré à la célébration d'une fête ${ }^{43}$. Des comptes annonçant le placement de ces fonds

39 P. Roussel, DCA, p. 241.

40 G. Roux, «Salles de banquet à Délos », BCH Suppl. I (1973), p. 525-554.

41 IG XI, 2, 287, $A, 1.85$.

42 Sur ces fêtes, cf. Ph. Bruneau, $C D H$, p. $342-343$.

43 Sur ce système, cf. Th. Homolle, «Comptes des Hiéropes du temple d'Apollon Délien », $B C H 6$ (1882), p. 112-113. 
par les hiéropes nous apprennent leur montant : le fond dit stèsileion est de 1500 drachmes, l'échénikeion de 3000 drachmes $^{44}$. Les fêtes portent le nom de leur fondateur. Parallèlement aux Aphrodisia officiels sont donc célébrés des Stèsileia et des Échénikeia qui se déroulent très probablement dans le sanctuaire fondé par la famille de Stèsiléos. Le revenu du stèsileion finance chaque année, outre une fête, la consécration d'un vase également dit stèsileion; il se peut que ces vases aient été dans un premier temps conservés dans l'oikos. Quant au revenu de l'échénikeion, il finance une fête. Lors des fêtes, un sacrifice a lieu ${ }^{45}$, peut-être suivi d'un banquct. À cette occasion, la statue d'Aphrodite offerte par Stèsiléos est nettoyée et parée aux frais des hiéropes.

Un passage d'un compte de 224 signale à la fois l'achat de torches pour les Aphrodisia et l'achat de feuilles d'or destinées à la statue d'Aphrodite ${ }^{46}$. Il est donc probable, si l'on s'en tient à l'hypothèse émise plus haut sur la statue, qu'en Hécatombaion avaient lieu à la fois la fête officielle des Aphrodisia, avec son chœur nocturne financé par les hiéropes, et les Stèsileia et les Échénikeia pour lesquels on redorait la statue offerte par Stèsiléos.

\section{Statut de l'Aphrodision de Stèsiléos}

La gestion de l'Aphrodision semble relever à la fois du sanctuaire d'Apollon et de la famille de Stèsiléos : dans le fonctionnement du sanctuaire de Stèsiléos interviennent les revenus des fonds déposés par Stèsiléos et Échénikè, mais aussi des sommes provenant de la caisse sacrée. L'entretien des bâtiments et de la statue est au moins partiellement financé par l'administration délienne. Les capitaux consacrés à la célébration des fêtes, en revanche, bien qu'ils soient gérés par les hiéropes, sont d'origine privée. L'organisation matérielle des fêtes n'apparaît jamais dans les comptes des hiéropes, contrairement à celle des Aphrodisia officiels; on peut supposer qu'elle est prise en charge par la famille de Stèsiléos.

L'existence d'inventaires de l'Aphrodision de Stèsiléos pendant la période de l'Indépendance, dont date la majorité des inscriptions de ce type que nous avons conservées, est discutable. De fait, le seul inventaire des hiéropes qui évoque un Aphrodision est très ambigu. Il s'agit d'un passage conservé dans son ensemble

\footnotetext{
44 ID 366, A, 1. 134 pour les Stèsileia; ID 287, A, 1. 122-123 pour les Echénikeia.

45 ID $366, A, 1.134$ pour les Stèsileia; ID $287, A, 1.123$ pour les Échénikeia.

46 ID $338, A a, 1.41-42$.
} 


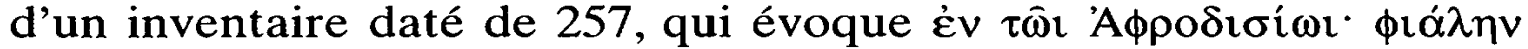

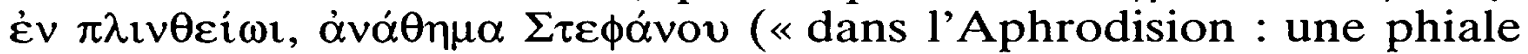
sur une base, offrande de Stéphanos $\gg)^{47}$. Deux éléments font douter que ce texte se rapporte à l'Aphrodision de Stèsiléos : d'abord, l'isolement de cette offrande serait curieux dans un sanctuaire où sont consacrés chaque année lors des Stèsileia un ou plusieurs vases; ensuite, la phiale de Stéphanos n'apparaît dans aucun des inventaires de l'époque athénienne, qui recensent pourtant des offrandes antérieures à 257 (statue de culte, statues de Diodotos et d'Échénikè, etc.). Je tends donc à penser que cet inventaire ne concerne pas le sanctuaire de Stèsiléos, mais l'Aphro-

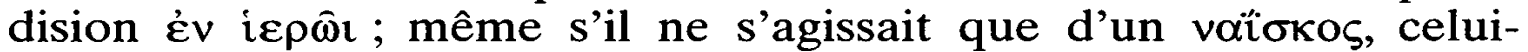
ci pouvait peut-être contenir une phiale isolée. Accepter cette hypothèse, c'est admettre que nous ne possédons aucun inventaire de l'Aphrodision de Stèsiléos datant de l'Indépendance. Cette absence, dans un corpus par ailleurs abondant, est notable : malgré les dangers du raisonnement a silentio, on pourrait y voir le signe que les offrandes déposées dans l'Aphrodision de Stèsiléos n'étaient pas gérées par les hiéropes, mais directement par le fondateur du sanctuaire et ses descendants.

La gestion du sanctuaire ne relève donc pas entièrement des hiéropes : une partie en revient à la famille du fondateur. La dimension familiale du culte est par ailleurs perceptible à plusieurs niveaux. La formulation des dédicaces des statues de Diodotos et d'Échénikè est remarquable en ce que le nom d'Aphrodite n'y figure pas; de même, les fêtes fondées par Stèsiléos puis par sa fille ne portent pas le nom de la déesse, mais privilégient l'onomastique familiale. La mainmise de la famille se fait sentir dans l'administration du sanctuaire au cours du $\mathrm{III}^{\mathrm{e}}$ siècle. Le fils du fondateur, Diodotos II, joue un rôle dans le culte d'Aphrodite : il est cité dans une loi malheureusement mal conservée qui réglemente apparemment les rapports entre les deux sanctuaires

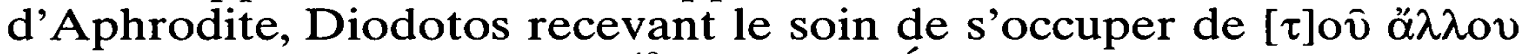
iєpov̄, «l'autre sanctuaire ${ }^{48}$. Sa sœur Échénikè fait des offrandes

$47 I G X I, 2,226, B, 1.6-7$. Cette phiale n'apparaît dans aucun autre inventaire. Sur la traduction de $\pi \lambda \mathrm{\imath v} \theta \varepsilon i \mathrm{ov}$ par «cadre», cf. M.-Chr. Hellmann, Recherches sur le vocabulaire de l'architecture grecque d'après les inscriptions de Délos, BEFAR 278 (1992), p. 340 , n. 3 ; ce sens me paraît peu vraisemblable ici.

48 IG $X I, 2,1029$ :

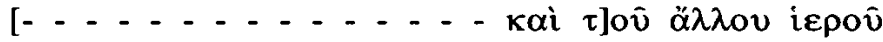

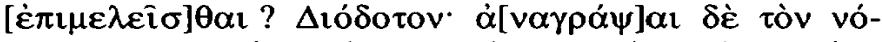

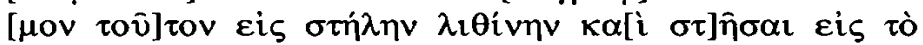

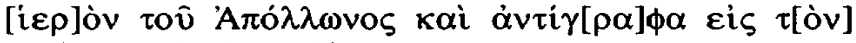

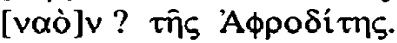


dans l'Aphrodision en 250, en même temps qu'elle consacre à Aphrodite une coupe d'argent et un vase d'or dans le sanctuaire d'Apollon; la même année, elle ajoute aux Stèsileia une deuxième fête de fondation, les Échénikeia. Une bonne part de la gestion du sanctuaire et de son enrichissement est assumée par la famille du fondateur.

Il semble donc que l'Aphrodision de Stèsiléos soit une sorte de chapelle familiale entretenue avec l'aide des hiéropes, mais probablement réservée, étant donné ses dimensions, à un public restreint. Il convient à présent de chercher à comprendre quel type de famille pouvait s'autoriser une manifestation de piété aussi originale et coûteuse, et dans quel but.

La famille de Stèsiléos est bien connue à Délos ${ }^{49}$. C'est une famille de notables dont plusieurs membres occupent pendant l'Indépendance des postes administratifs et honorifiques importants. Stèsiléos lui-même est archonte en 305, chorège des Apollonia en 284 et chorège des Dionysia en $280^{50}$; ces chorégies le désignent comme un notable fortuné. Son fils Diodotos est hiérope en $286^{51}$. L'arrière-petit-fils du fondateur, Stèsiléos III, est archonte en $207^{52}$.

La fondation instituée et entretenue par cette famille est d'une nature tout à fait originale. Au lieu de consacrer une offrande magnifique dans l'Aphrodision év i $\varepsilon \rho \hat{\omega}$, Stèsiléos fait choix de doubler l'Aphrodision officiel d'un nouvel édifice construit en dehors du sanctuaire d'Apollon. Ce faisant, il délaisse une statue offerte par Thésée pour consacrer une nouvelle Aphrodite délienne et abandonne un lieu de culte où $\Lambda$ thènes a été omniprésente pendant un siècle et demi pour installer un nouveau culte dans la ville. Le principal modèle qu'a pu suivre ici Stèsiléos, c'est celui de l'Archégésion, ce sanctuaire isolé réservé aux seuls Déliens; mais alors que l'Archégésion est à l'écart de toutes les autres constructions, l'Aphrodision de Stèsiléos s'installe sinon en bordure du principal quartier d'habitation, du moins à sa proximité.

Les choix architecturaux qui président à la construction de l'Aphrodision ne sont pas non plus banals. Le plan rectangulaire du temple est exceptionnel ; son appareil se distingue des construc-

\footnotetext{
49 Voir Cl. Vial, loc. cit.

so $I G X I, 2,1067, b, 1.5$ pour l'archontat; $I G X I, 2,105,1.4$ pour la chorćgic des Apollonia; $I G X I, 2,107,1.13$ pour la chorégie des Dionysia.

51 ID 155, b, 1. 7.

52 ID $366, A, 1.87$
} 
tions déliennes classiques. L'appareil de l'oikos en revanche rappelle des constructions cycladiques comme l'oikos des Naxiens et l'orientation du temple et de l'autel semble calquée sur le modèle archaïque de l'Héraion. Deux tendances se dessinent : d'une part la recherche de modèles architecturaux nouveaux, d'autre part l'imitation de modèles déliens antérieurs à l'époque de la première Amphictyonie.

L'ensemble donne l'impression que la fondation de Stèsiléos cherche à se démarquer des habitudes déliennes de l'époque de l'Amphictyonie, aussi bien par le choix de la destinataire du culte que par la décision de s'éloigner du sanctuaire d'Apollon, pour se rapporter à des modèles archaïques antérieurs à la présence athénienne et établir à partir de ces modèles un nouveau type d'établissement religieux qui réponde à une évolution des mentalités. Ne peut-on voir dans ces choix - refus des modèles de l'époque athénienne, éloignement du sanctuaire panhellénique, imitation de traditions locales anciennes, dimension familiale - l'expression d'un sentiment d'appartenance à une communauté proprement délienne qui s'accorderait assez bien au contexte historique de la fondation et aux caractéristiques sociales du fondateur?

L'Aphrodision de Stèsiléos se révèle donc être un sanctuaire exceptionnel par son statut mixte et sa dimension familiale. Le culte d'Aphrodite y sert à manifester la puissance d'une famille autant que sa révérence envers la déesse; par certains aspects, il paraît présenter un caractère politique.

\section{NOUVElle PRATIQUE OfFICIELlE : APHRODITE PARMI LES ÉTRANGERS}

Avec l'installation des Athéniens en 167 et le changement de population et d'administration qui en résulte, la famille de Stèsiléos disparaît des inscriptions déliennes, probablement expulsée de l'île. On pourrait s'attendre à ce que le sanctuaire de Stèsiléos périclite après le départ de ses fondateurs. C'est le contraire qui se produit : les inscriptions mentionnant l'Aphrodision se multiplient tandis que le sanctuaire s'agrandit et s'enrichit d'offrandes nouvelles.

\section{Évolution du sanctuaire}

L'évolution du sanctuaire peut être perçue à la fois à travers les vestiges et dans les inscriptions. 
Les vestiges révèlent qu'au noyau initial du sanctuaire sont ajoutées pendant la période athénienne au moins quatre nouvelles constructions à une seule pièce. Ces constructions, numérotées d'Ouest en Est de 2 à 5 (Fig. 2), prennent appui les unes sur les autres. Les murs Est-Ouest de l'édifice 2 s'appuient sur le mur Ouest de l'édifice 3; le mur Nord des édifices 3 et 4 est continu; le mur de façade de l'édifice 4 s'appuie sur le mur Nord de l'édifice 5. L'édifice 5 est donc le plus ancien; il est construit dans un appareil assez voisin de celui de l'oikos 1, mais de bien moindre qualité (Fig. 8); des blocs de granit ou de gneiss y remplacent le marbre. Il est suivi par les édifices 3 et 4 , qui datent d'une même phase de construction, puis par l'édifice 2.

Les édifices 3, 4 et 5, dont l'orientation principale suit celle de la rue qui quitte le sanctuaire et se prolonge vers l'Est, sont construits sur une surface plane dont le niveau est plus haut que celui de la cour du temple : le changement de niveau se fait le long de la ligne que l'on peut tracer entre la première marche de l'escalier qui monte vers la rue Est et le mur Ouest de l'édifice 3. Les terres de la partie orientale du sanctuaire sont retenues au Nord par le mur Ouest de l'édifice 3, au Sud par un mur situé dans le même alignement, mais qui n'est conservé qu'en fondation. Entre ces deux murs, une rampe mène de la cour du temple à la partie orientale du sanctuaire. L'édifice 2 se trouve à un niveau intermédiaire entre la cour du temple et l'ensemble oriental; ses dimensions sont réduites, son appareil médiocre : selon toute apparence, c'est une pièce rapportée ajoutée après coup à ce programme de construction.

Si je développe cette description, c'est pour montrer que les travaux d'extension du sanctuaire qui datent de cette deuxième phase d'utilisation ne sont pas mineurs : il a fallu remblayer l'ensemble de la zone située à l'Est du temple pour pouvoir y construire cette extension. La différence d'orientation entre la cour du temple et la partie orientale du sanctuaire laissait penser que les deux ensembles ne dataient pas de la même phase de construction; un sondage que nous avons réalisé en 2005 dans le plus ancien des édifices ${ }^{53}$ a permis de dater le remblai de la deuxième moitié du deuxième siècle.

Le passage de la période de l'Indépendance à la période athénienne est marqué pour nous par une modification radicale de la documentation écrite. Nous n'avons plus désormais de comptes

${ }^{53}$ Le rapport des fouilles paraîtra dans le $B C H 130.2$ (2006). 
d'entretien, non que l'entretien cesse d'être assuré par les gestionnaires du sanctuaire d'Apollon, mais simplement parce que les comptes ne sont plus gravés après 140 . Nous disposons en revanche de deux séries d'inventaires évoquant le sanctuaire au milieu du $\mathrm{II}^{\mathrm{e}}$ siècle; cinq des huit textes conservés datent des environs de 156 , les trois autres des environs de 140.

L'inventaire le mieux conservé est celui de l'année 156 (ID 1417, $A$, II, 1. 1-21). En voici le texte :

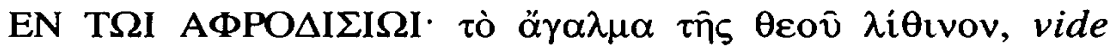

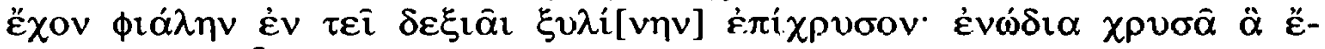

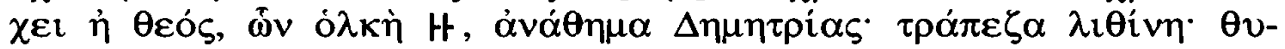

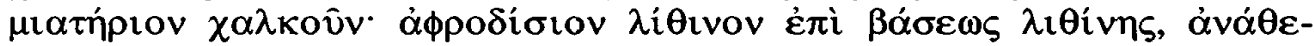

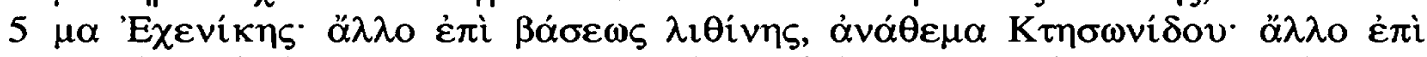

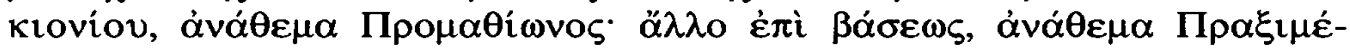

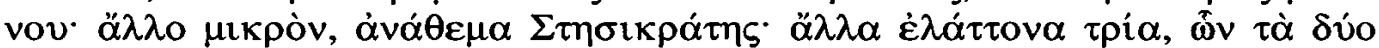

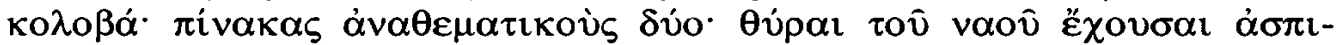

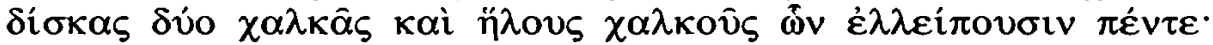

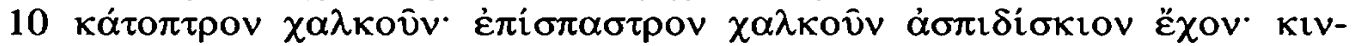

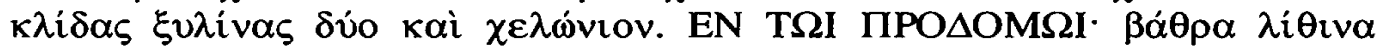

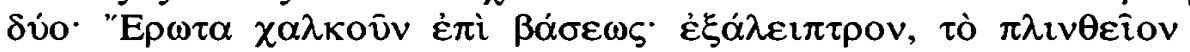

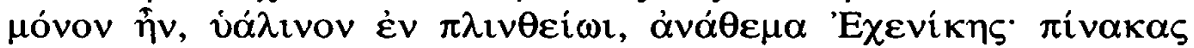

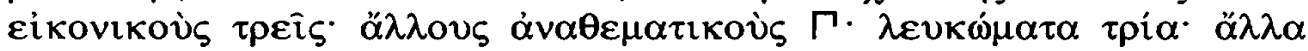

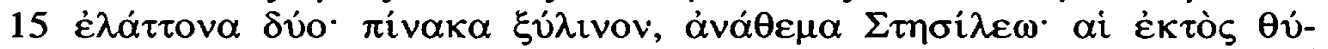

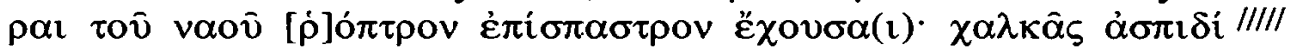

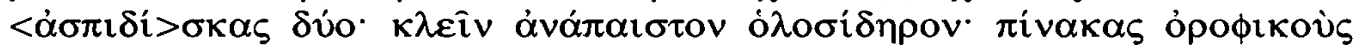

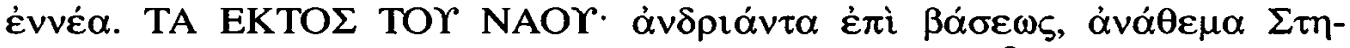

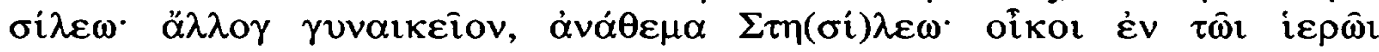
$\tau \varepsilon \theta \nu \rho \omega \mu \varepsilon ́$ vor $\kappa \varepsilon \rho \alpha-$

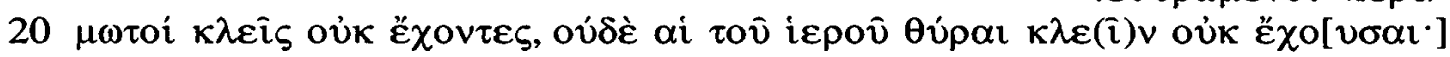

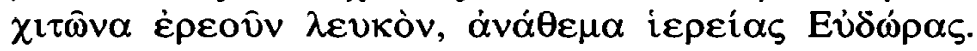

«DANS L'APHRODISION : la statue de culte de la déesse, en marbre, tenant dans la main droite une phiale de bois doré; des boucles d'oreilles d'or que porte la déesse, d'un poids de 2 drachmes, offrande de Dèmètria; une table de marbre; un encensoir de bronze; une statue d'Aphrodite en marbre sur une base de marbre, offrande d'Échénikè ; une autre sur une base de marbre, offrande de Ctèsonidès; une autre sur une colonnette, offrande de Promathion; une autre sur une base, offrande de Praximénès; une autre petite, offrande de Stèsicratè ; trois autres plus petites, dont deux mutilées; deux tableaux votifs; les portes du naos avec deux disques de bronze et des clous de bronze dont cinq manquent ; un miroir de bronze; un heurtoir avec un petit disque de bronze; deux grilles de bois et un verrou. DANS LE PRODOMOS : deux bancs de marbre; un Éros de bronze sur une base; un vase à parfum (la base seule restait) de verre dans une base, offrande d'Échénikè ; trois portraits ; 5 tableaux votifs; trois tablettes blanchies; deux autres plus petites; un tableau de bois, offrande de Stèsiléos; les portes extérieures du temple avec une poignée-heurtoir; deux disques de bronze; une clef entièrement de fer forgé ; neuf caissons peints. À L'EXTÉRIEUR DU TEMPLE : une statue d'homme sur une basc, offrande de Stèsiléos ; une autre de femme, offrande 
de Stèsiléos; des oikoi dans le sanctuaire, munis de portes, couverts de tuiles, sans clef; les portes du sanctuaire n'ayant pas non plus de clef; un chiton de laine blanc, offrande de la prêtresse Eudôra. »

C'est ce passage, avec un autre de la même série ${ }^{54}$, qui nous fournit la description de la statue de culte de Stèsiléos citée plus haut. L'ensemble du sanctuaire est évoqué dans cet inventaire, qui le divise en trois ensembles : naos, prodomos et extérieur du temple. Les oikoi mentionnés à l'extérieur du temple sont très probablement les édifices décrits plus haut. Leur nombre est précisé dans un inventaire de quelques années plus tardif dont le rédacteur a recensé cinq oikoi ${ }^{55}$. Les offrandes dont nous connaissons les dédicataires datent de l'époque de l'Indépendance : Stèsiléos, Échénikè, Ctèsonidès et Praximénès et sont des Déliens de l'Indépendance ${ }^{56}$.

Les inventaires des années suivantes ${ }^{57}$ mentionnent de nouvelles offrandes, que l'on peut attribuer avec certitude à l'époque athénienne : apparaissent par exemple deux encensoirs de marbre et un de bronze, trois paires de souliers votifs, trois petites figurines d'animaux en bois, deux ou trois chitons consacrés, un chiton d'enfant en laine, une toge, un éventail, un petit bas-relief portant une colombe, un objet d'or d'un poids d'une obole, trois nouvelles paires de sandales consacrées; quelques années plus tard, on offre encore à la déesse un chiton de laine, un autre chiton, un objet de cristal et un portrait. Ces offrandes nouvelles forment un ensemble composite et assez modeste.

Les grands travaux d'extension du sanctuaire réalisés au milieu du II $^{\mathrm{e}}$ siècle et le dépôt d'offrandes nouvelles montrent que la fondation de Stèsiléos, loin de dépérir après la disparition de la famille de son fondateur, connaît pendant la période athénienne une fréquentation accrue. Si l'installation de nouveaux bâtiments laisse penser que le public est plus nombreux qu'à l'époque de l'Indépendance, la nature des offrandes montre qu'il est aussi d'une moindre aisance.

54 ID 1423, $B a$, II , 1. 17-23. La première série des inventaires athéniens de l'Aphrodision regroupe cinq textes très semblables répartis autour de l'année 156 av. J.-C. : ID 1412 , $a$, l. $28-35$; ID $1414, a$, II, I. $1-18$; ID $1417, A$, II, $1.1-21$; ID 1423, Ba, II, 1. $17-23 ; I D$ 1426, $B$, II, 1. 2-22.

55 ID $1426, B$, II, 1. 20-21 (entre 156 et 146 av. J.-C.).

56 Cf. Cl. Vial, Délos indépendante, BCH Suppl. X (1984), p. 157 et 135 ou 256.

57 Le mieux conservé des inventaires de cette deuxième série est $I D 1442, B, 1.30$ 35, daté de l'archontat de Métrophanès, c'est-à-dire de 146 ou 145 av. J.-C. L'inscription $I D$ 1440, $A, 1$. 1-6 est un peu antérieure; l'inscription ID 1443, $B$, II, 1. 92-100 date d'cntrc 145 et 141 . 


\section{Le culte}

Les comptes nous faisant défaut, la célébration des Stèsileia et des Échénikeia n'est pas davantage attestée à l'époque athénienne que celle des Aphrodisia officiels; quant aux inventaires, ils sont trop lacunaires pour permettre de savoir si les vases des Stèsileia continuent à être consacrés chaque année.

Les vestiges en revanche donnent des indices assez sûrs. Si le sanctuaire est étendu au milieu du $\mathrm{II}^{\mathrm{e}}$ siècle, c'est pour accueillir du public; il est donc vraisemblable que la célébration des fêtes continue. Cette hypothèse est renforcée par les résultat des sondages effectués en 2005 dans l'oikos 5 : nous y avons mis au jour un matériel exclusivement culinaire (vaisselle, amphores et fragments de réchauds), en grande quantité. La célébration de banquets dans l'Aphrodision, que nous ne pouvons que supposer pour la période de l'Indépendance sur la foi d'inscriptions mentionnant des sacrifices, est donc très probable à l'époque athénienne.

Alors que le personnel cultuel de l'époque de l'Indépendance nous est totalement inconnu, les inventaires athéniens transmettent les noms de trois prêtresses d'Aphrodite, Eudôra ${ }^{58}$, Pleistarchè $^{59}$ et Iônis ${ }^{60}$, mais ces noms sont inconnus par ailleurs et ne nous permettent donc pas de savoir dans quelle catégorie de population les prêtresses d'Aphrodite étaient recrutées. Seule Eudôra, qui offre avant 156 un chiton de laine blanc dans l'Aphrodision de Stèsiléos, apparaît dans un autre contexte dans les inscriptions de Délos : peut-être est-ce la même Eudôra qui fait don de trois phiales dans le Sarapieion avant $146^{61}$.

\section{Statut de l'Aphrodision}

Le sanctuaire a perdu à l'époque athénienne son statut semiprivé. L'existence d'inventaires annuels indique que les biens de l'Aphrodision sont désormais contrôlés par les gestionnaires athéniens; l'accroissement du sanctuaire montre que les restrictions d'accès que l'on peut supposer pour la période de l'Indépendance ne sont plus appliquées à l'époque athénienne; la dimension familiale enfin a complètement disparu avec la famille, et l'on ne sait même pas si les fêtes familiales persistent sous leur nom et leur forme d'origine.

\footnotetext{
58 ID 1412, $a$, 1. 35 ; 1414, a II, 1. $18 ; 1417, A$ II, 1. $21 ; 1426, B$ II, 1. 13.

59 ID 1423, Ba II, l. $19 ; 1426, B$ II, l. 5.

60 ID 1442, B, 1. 33.

61 ID 1442, A, 1. 63.
} 
Les dernières inscriptions conservées concernant l'Aphrodision datent de la fin du $\mathrm{II}^{\mathrm{e}}$ siècle. Il s'agit de deux stèles découvertes en remploi l'une à proximité du sanctuaire d'Apollon, dans une église installée à l'intérieur du portique de Philippe, l'autre à proximité du sanctuaire d'Aphrodite, dans le mur de Triarius au Nord de la Maison de l'Hermès ${ }^{62}$. Les deux inscriptions diffèrent légèrement, mais commémorent toutes deux des réparations, voire des restaurations opérées dans un sanctuaire d'Aphrodite par un nommé Dionysios, fils de Nicôn, du dème de Pallène, qui a été épimélète à Délos en $110^{63}$. Elles sont intéressantes du point de vue du statut du sanctuaire. Dionysios finance personnellement

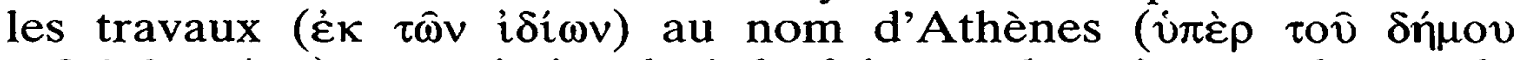

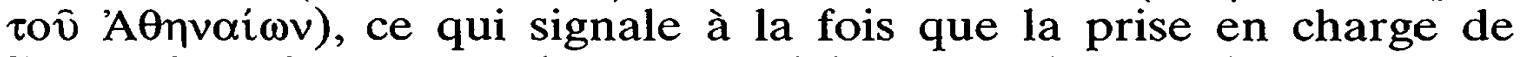
l'entretien des sanctuaires secondaires par les gestionnaires du sanctuaire d'Apollon s'est affaiblie et que la dimension proprement délienne de l'Aphrodision a bel et bien disparu. Mieux encore, Dionysios présente son intervention comme une sorte de nouvelle fondation. L'une des inscriptions ${ }^{64}$ annonce que

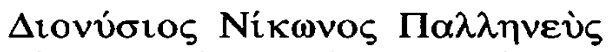

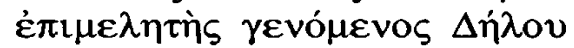

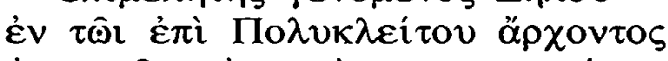

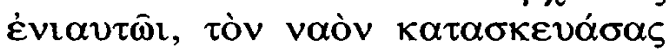

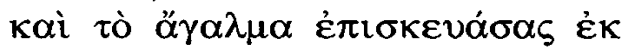

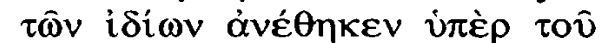

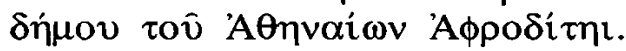

«Dionysios fils de Nicôn, du dème de Pallène, ayant été épimélète de Délos pendant l'année de l'archontat de Polycleitos, ayant reconstruit le temple et réparé la statue sur ses propres fonds, les a dédiés au nom du peuple d'Athènes à Aphrodite ».

62 ID 1810 et 1811 ; sur l'emplacement de découverte et l'attribution de ces inscriptions à l'Aphrodision de Stèsiléos, cf. Ph. Bruneau, $C D H$, p. 338-339. L'emploi du terme vaòv dans la première inscription et la mention de bases dans la seconde incitent à les rapporter toutes deux à l'Aphrodision de Stesiléos. Nous le faisons toutefois sans certitude. Il se peut que Dionysios ait voulu publier la commémoration de ses bienfaits en les affichant à la fois sur le lieu de ses travaux et dans le sanctuaire d'Apollon, certainement plus fréquenté que celui d'Aphrodite; mais si l'inscription découverte dans le portique de Philippe est un doublet de celle du mur de Triarius, il est curieux que le texte ne soit pas tout à fait le même. Il se peut aussi que Dionysios ait fait des réparations dans les deux sanctuaires d'Aphrodite; mais l'inscription découverte à proximité du sanctuaire d'Apollon, qui devrait alors logiquement se rapporter à l'Aphrodision èv i $\varepsilon \rho \hat{\omega}$, cite des bases dont nous n'avons aucune autre mention pour ce sanctuaire.

${ }^{63}$ Sur ce personnage, voir P. Roussel, $D C A$, p. 109. Dionysios est connu à Délos par son statut d'épimélète et par de nombreuses offrandes à divers dieux (ID 1812, $1813,1814,2011$ bis), en particulier une chapelle dans le sanctuaire des dieux syriens (ibid., p. 258;ID 2221).

64 ID 1810. 
L'autre inscription ${ }^{65}$ mentionne que

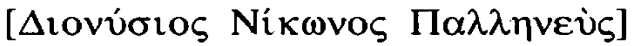

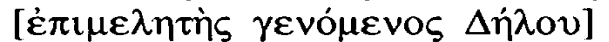

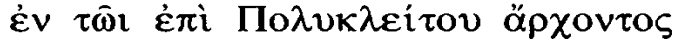

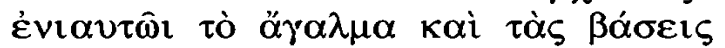

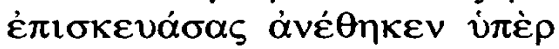

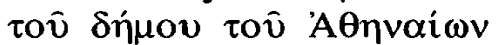

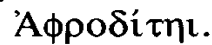

«Dionysios fils de Nicôn, du dème de Pallène, épimélète de Délos pendant l'année de l'archontat de Polycleitos, ayant réparé la statue et les bases, les a dédiées au nom du peuple d'Athènes à Aphrodite ».

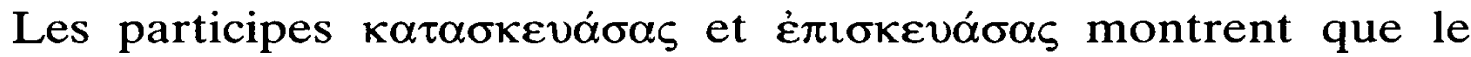
sanctuaire devait être en piètre état avant l'intervention de Dionysios ; l'emploi du terme ơvé $\theta \eta \kappa \varepsilon v$ présente cet Aphrodision restauré comme une offrande de l'Athénien et fait complètement disparaître la figure du premier fondateur.

Se dessinent donc à travers les inscriptions et les vestiges deux phases d'utilisation bien distinctes du même sanctuaire, où l'on s'étonne de la continuité du culte et de la gestion malgré le changement complet de public et de «propriétaires».

\section{CONCLUSION}

Le culte d'Aphrodite n'est assurément pas un culte majeur à Délos; la déesse appartient toutefois à un groupe de divinités secondaires bien représentées et ancrées dans les traditions anciennes des cultes déliens. Si l'on en sait peu sur le culte officiel d'Aphrodite dans le sanctuaire d'Apollon, c'est probablement qu'il occupait une place modeste dans la hiérarchie des cultes déliens qui apparaît à travers l'abondance des documents conservés. En revanche, si l'on en sait tant sur l'Aphrodision de Stèsiléos, c'est vraisemblablement parce qu'il s'agit d'un lieu de culte relativement original, du moins dans un premier temps pour un public restreint auquel le culte d'Aphrodite sert de prétexte et qui fait de ses manifestations de piété un instrument de grandeur familiale, créant ainsi un culte dont la forme et les enjeux sont nouveaux à Délos. Dans une deuxième phase, l'Aphrodite de Stèsiléos perd ses liens avec son fondateur pour devenir l'objet d'un culte populaire, au même titre que les dieux étrangers dont les sanctuaires sont rendus publics à la même époque.

65 ID 1811. 
Le culte d'Aphrodite est donc assez complexe à Délos en ce qu'il revêt trois formes distinctes. Les deux formes publiques sont intéressantes sans être exceptionnelles : les Aphrodisia sans doute liés à l'ancien xoanon conservé dans le sanctuaire d'Apollon sont une fête délienne parmi d'autres du même type; quant au culte public rendu à l'époque athénienne dans l'Aphrodision fondé par Stèsiléos, il semble tout à fait parallèle aux cultes étrangers pratiqués dans le quartier voisin. Le culte rendu à l'Aphrodision pendant l'Indépendance est en revanche d'une grande originalité : non seulement il s'agit d'un cas de fondation privée particulièrement bien documenté, mais le culte pratiqué ici est un culte parallèle au culte officiel, ce qui le distingue des cultes privés étrangers rendus à Délos par des populations non grecques, qui suppléent à l'absence de dieux étrangers du panthéon délien officiel. Le grand intérêt du culte fondé par Stèsiléos, c'est qu'il relève autant de la politique que de la religion, et qu'il constitue une innovation à forte dominante archaïsante, traditionnelle et régionaliste ; toutes ces dimensions disparaissent après 167 pour laisser place à un culte beaucoup plus banal, adressé à un public qui fréquente le temple d'Aphrodite comme ceux de Sarapis ou d'Isis.

Cécile DuRvYE

École française d'Athènes. 
Fig. 1. - Délos. Relevé des zones fouillées en 1970 (d'après (C) EFA, 7240 bis). 


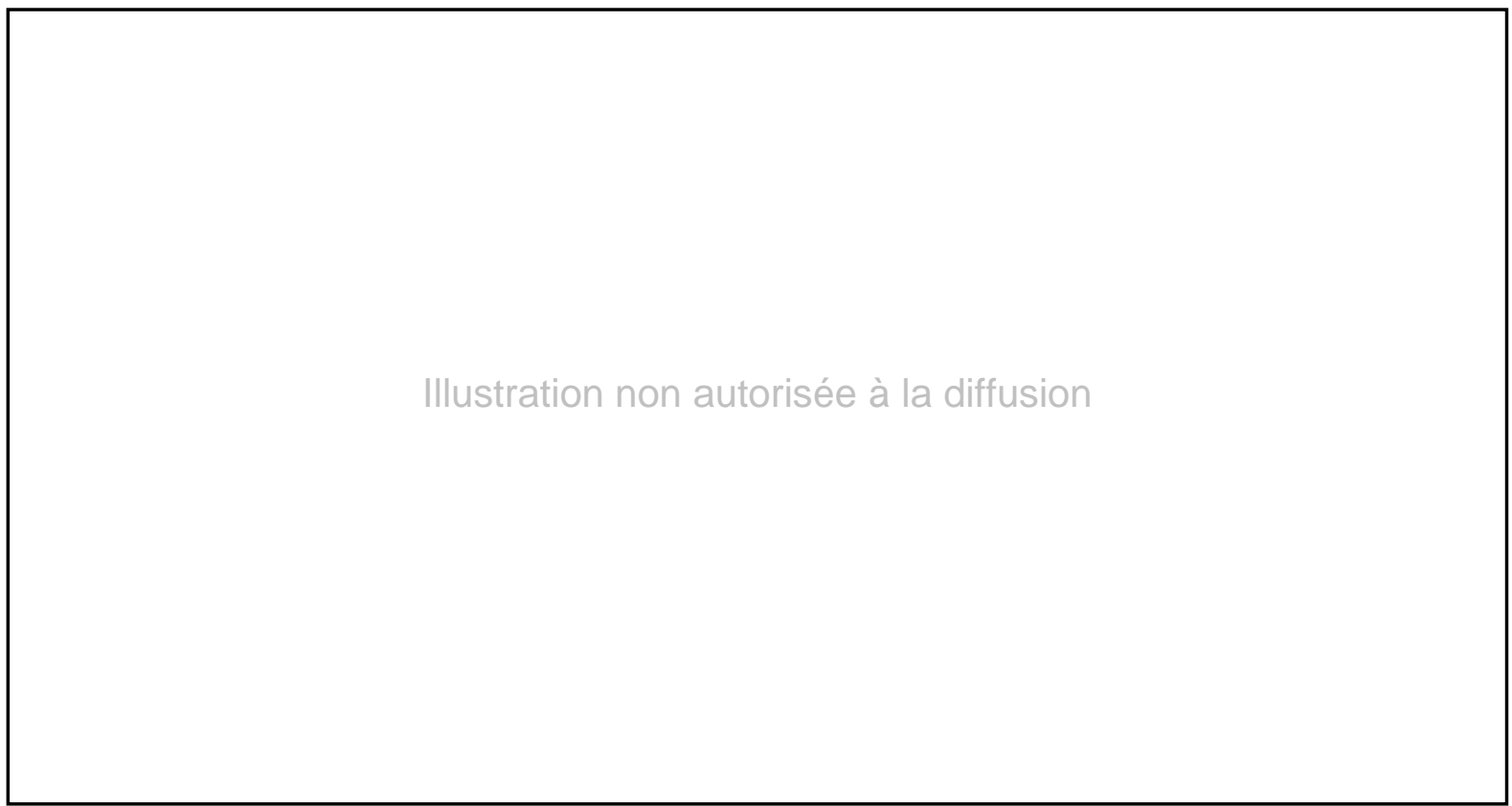

Fig. 3. - Temple, autel et oikos 1 . Vue prise vers le Nord-Ouest (C. Durvye, (1) EFA). 


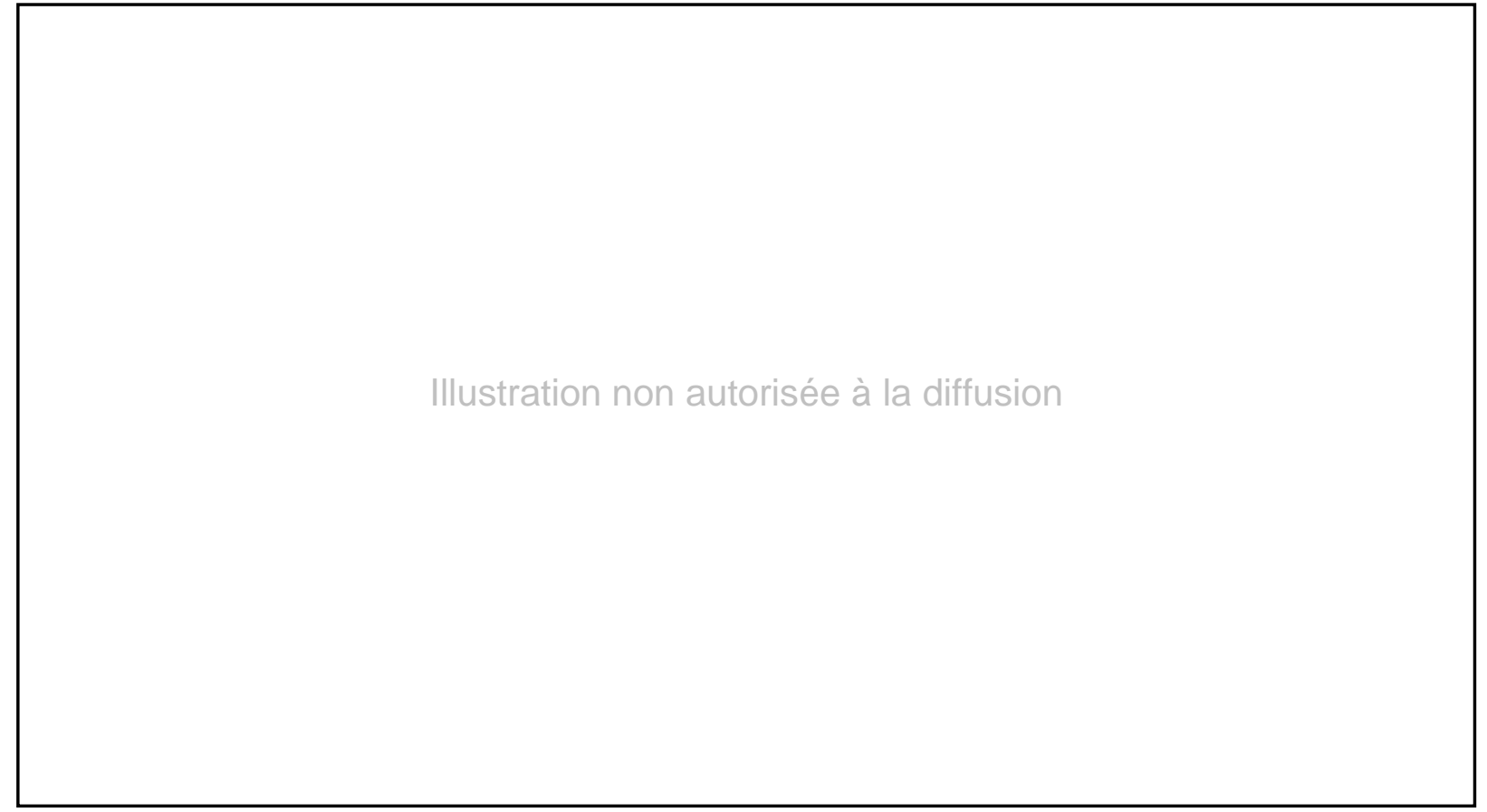

Fig. 5. - Parement extérieur du mur Est du temple (C. Durvye, (C) EFA).

Fig. 6. - Autel. Vue prise vers le Nord (C. Durvye, (C) EFA). 
Fig. 7. - Parement extérieur du mur Est de l'oikos 1

(C. Durvye, (C) EFA).

Fig. 8. - Parement intérieur du mur Sud de l'oikos 5

(C. Durvye, () EFA). 